Thermal Design and Performance of the Gamma-Ray Spectrometer for the MESSENGER Spacecraft

M. Burks, C. P. Cork, D. Eckels, E. Hull, N. W. Madden, W. Miller, J. Goldsten, E. Rhodes, B. Williams

October 20, 2004

IEEE, 14th International Workshop on Room-Temperature Semiconductor X- and Gamma-Ray Detectors Rome, Italy

October 16, 2004 through October 22, 2004 
This document was prepared as an account of work sponsored by an agency of the United States Government. Neither the United States Government nor the University of California nor any of their employees, makes any warranty, express or implied, or assumes any legal liability or responsibility for the accuracy, completeness, or usefulness of any information, apparatus, product, or process disclosed, or represents that its use would not infringe privately owned rights. Reference herein to any specific commercial product, process, or service by trade name, trademark, manufacturer, or otherwise, does not necessarily constitute or imply its endorsement, recommendation, or favoring by the United States Government or the University of California. The views and opinions of authors expressed herein do not necessarily state or reflect those of the United States Government or the University of California, and shall not be used for advertising or product endorsement purposes. 


\title{
Thermal Design and Performance of the Gamma-Ray Spectrometer for the MESSENGER Spacecraft
}

\author{
M. Burks, C. P. Cork, D. Eckels, E. Hull, N.W. Madden, W. Miller, J. Goldsten, E. Rhodes, B. Williams
}

\begin{abstract}
A gamma-ray spectrometer (GRS) has been built and delivered to the Mercury MESSENGER spacecraft which launched on August 3, 2004, from Cape Canaveral, Florida. The GRS, a part of seven scientific instruments on board MESSENGER, is based on a coaxial high-purity germanium detector. Gamma-ray detectors based on germanium have the advantage of providing excellent energy resolution, which is critical to achieving the science goals of the mission. However, germanium has the disadvantage that it must operated at cryogenic temperatures (typically $\sim 80 \mathrm{~K}$ ). This requirement is easy to satisfy in the laboratory but difficult near Mercury, which has an extremely hot thermal radiation environment. To cool the detector, a Stirling cycle mechanical cooler is employed. In addition, radiation and conduction techniques are used to reduce the GRS heat load. Before delivering the flight sensor, a complete thermal prototype was built and tested. The results of these tests, including thermal design, radiative and conductive heat loads, and cooler performance are described.
\end{abstract}

\section{INTRODUCTION}

$\mathrm{M}$ ESSENGER (MErcury Surface, Space ENvironment, GEochemistry, and Ranging) is the first mission to Mercury since Mariner 10 made three fly-bys in the mid-1970s. It will also be the first mission to enter Mercury orbit. MESSENGER has seven scientific instruments on board, giving it a full range of spectroscopic capabilities including IR, UV, $\mathrm{x}-$ ray, gamma-ray, neutron and charged-particle spectroscopy. It will also have a magnetometer, visible imaging camera, radio science, and a laser altimeter. Some of the key science questions to be addressed include understanding Mercury's surface composition, studying its magnetic field, and verifying the suspected presence of ice at the poles.

On August 3, 2004, the MESSENGER spacecraft launched from Cape Canaveral, Florida, on a Boeing Delta II rocket. The spacecraft will reach Mercury orbit in March 2011. Along the way it will make several flybys: one of Earth, two of Venus, and three of Mercury itself. These flybys will serve the purpose of losing the energy gained while traveling towards the Sun. Finally, it will insert into a 12-hour orbit around the planet. The orbit will be highly eccentric with the apoapsis being $15,000 \mathrm{~km}$ and periapsis being only $200 \mathrm{~km}$ from the surface of the planet. The mission will last one Earth year ( $\sim$ four Mercury years).

Manuscript received October 13, 2004. This work was performed under the auspices of the U.S. Department of Energy by University of California Lawrence Livermore National Laboratory under contract No. W-7405-Eng-48.

M. Burks (email: burks@llnl.gov), C. P. Cork, D. Eckels, E. Hull, N.W. Madden, W. Miller are with Lawrence Livermore National Laboratory.

J. Goldsten, E. Rhodes, B. William are with the Johns Hopkins University Applied Physics Laboratory.
Mercury has little atmosphere and a weak magnetic field, and is therefore largely unshielded from cosmic and solar radiation. The gamma-ray spectrometer (GRS), one component of the MESSENGER Gamma-Ray and Neutron Spectrometer (GRNS) instrument, will record the characteristic gamma rays emitted from surface elements as a result of reactions with cosmic rays and, in conjunction with the other spectrometers, help determine the surface composition. The spacecraft and science objectives of the mission are described in [1]-[3].

This work focuses on the gamma ray spectrometer (Fig. 1). The energy band of interest for the GRS includes gamma rays from $100 \mathrm{keV}$ to $10 \mathrm{MeV}$. Originally, it was conceived that a $\mathrm{NaI}$ scintillator would fly as the gamma-ray spectrometer. NaI has the advantage of high atomic number and thus good stopping power. However, it was determined that the limited resolution of a scintillator would make it difficult to achieve the desired science objectives. In contrast, a germanium-based spectrometer gives superior resolution but has the distinct liability that it must operate at cryogenic temperatures. At the outset, it was not clear that this would be possible due to the harsh thermal environment found at Mercury. Therefore, a feasibility testing program was undertaken to determine if a germanium spectrometer could be flown. The main objectives were to determine a suitable cooling scheme as well as to implement a design that would minimize the GRS thermal burden.

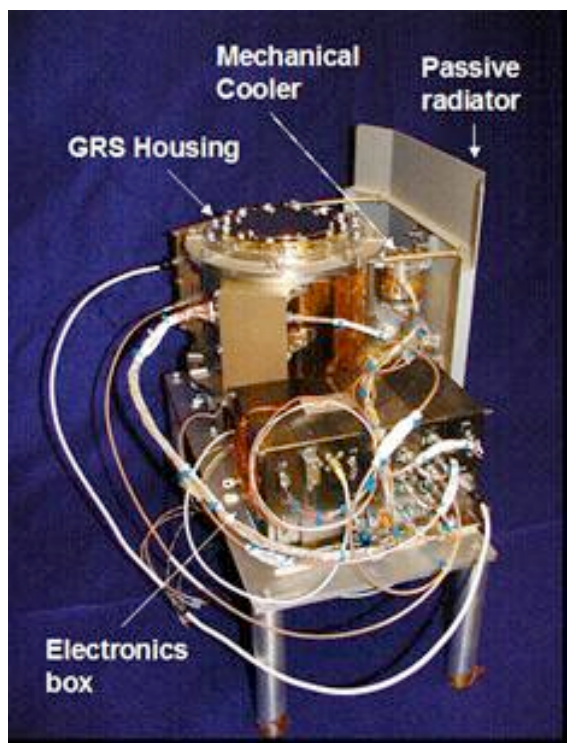

Fig. 1. Assembled gamma-ray spectrometer before delivery to the spacecraft. The GRS housing is shown along with the mechanical cooler. The cooler is mounted externally with the cold finger penetrating into the housing. The passive radiator dissipates waste heat from the cooler. 
There are two approaches to cooling a germanium detector in space. The first is to use passive radiative cooling. This method relies on the GRS being able to view the cold background of deep space. However, this solution is not viable in Mercury's orbit because of the hot thermal radiation environment. The second approach is to use mechanical cryocooling. However, there is limited experience with this approach for germanium detectors in space [4]. Furthermore, there are a limited number of choices of mechanical cryocoolers that provide the heat lift needed with the required specifications of low mass, low input power, and long lifetime. Therefore, as part of this program, a suitable mechanical cooler was selected, integrated into the GRS, and fully tested.

A brief overview of the GRS is given followed by a detailed discussion of the thermal design and testing of the system.

\section{GAMMA-RAY SPECTROMETER}

Two versions of the GRS were delivered to undergo testing. The first was the engineering model and the second was the flight unit. The engineering model also served as a backup if it had been needed.

\section{A. Germanium Detector and Encapsulation}

The GRS is based on a high-resolution coaxial germanium detector. The crystal is $50 \mathrm{~mm}$ in diameter by $50 \mathrm{~mm}$ in length with a thin stable entrance window ( 0.2 microns $)$. It has an efficiency of $25 \%$ (relative to a $3 " \times 3 " \mathrm{NaI}$ detector) at 1.33 $\mathrm{MeV}\left({ }^{60} \mathrm{Co}\right)$. The detector is a high depletion voltage N-type which was chosen for its radiation damage resistance and annealing capabilities (see below). The detector is rigidly clamped (44-G preload) and sealed inside a valve-less hermetic encapsulation [5]. The encapsulation is pressurized to 3 bar with clean dry nitrogen. The encapsulation is cooled by the mechanical cooler via a flexible copper braid, which employs electrical isolation to prevent cooler electronic noise from interfering with the detector signal.

\section{B. Kevlar Suspension}

A suspension system was designed in order to allow the detector encapsulation to withstand the launch on the Delta II launch vehicle. The suspension was designed to handle up to 44 G's. It was also necessary for the suspension system to provide thermal isolation between the GRS housing and detector. Therefore, Kevlar (Dupont 29) was chosen for its high tensile strength and low thermal conductivity [6]. The Kevlar fiber suspension system held the detector to a frame and provided support and thermal isolation with a combined criss-cross and annular stringing pattern.

A method was devised for measuring frame deformation to insure that tension on the cord was both properly applied and maintained long term. A test unit was strung and cord tension measured by this means for one year with no measurable creep or change in frame deflection.

\section{Electronic Readout}

A rugged internal signal contact brings the detector signal out from the hermetic encapsulation to a charge-sensitive preamplifier. The preamplifier is operated with an uncooled junction field effect transistor (JFET) and resistive feedback. In addition, a charge-reset circuit is used for large overloads due to cosmic ray events and helps signal detection rate. Following the preamplifier is a bipolar shaper (6 microsecond peaking time).
The bipolar signal is then sampled by a high-speed analog-todigital converter (ADC) and fed into a field programmable gate array (FPGA) where another shaping step is performed. The FPGA shaping stage combined with the analog shaping is necessary to minimize the effects of the cyrocooler microphonics on the detector energy resolution. The measured resolution of the installed flight GRS is $3.5 \mathrm{keV}$ full-width halfmaximum (FWHM) at $1.33 \mathrm{MeV}$ with the cryocooler operating.

\section{Mechanical Cooler}

A Ricor K508 Stirling-cycle cryocooler cools the detector. The nominal detector operating range is 85 to $90 \mathrm{~K}$. The input power required for the cooler ranges from 10 to $15 \mathrm{~W}$. The cooler operates at a constant $15 \mathrm{~V}$. Pulse width modulation and temperature feedback are used to control the detector to $+/-0.03$ K. As described below, the cooler rejects its waste heat to the passive radiator as well as to the body of the GRS housing. The Ricor has a maximum operating temperature of $85{ }^{\circ} \mathrm{C}$, and thus the body of the GRS must remain below this temperature for the cooler to function. In addition to the cooler testing and characterization described below, a customization and selection process was developed with the manufacturer for hand selecting the coolers for flight.

\section{E. Radiation Shields}

Due to the limited heat lift capability of the mechanical cooler, it was necessary to limit the thermal burden to the detector. The Kevlar suspension system limited the conductive load. However, a significant heat load due to infrared radiation remains. Therefore, several concentric infrared radiation shields were employed. A description of these shields and their effectiveness is given below.

\section{THERMAL ENVIRONMENT}

One of the main challenges in designing the GRS was to ensure the operation of the detector at $\sim 85 \mathrm{~K}$ given the harsh thermal environment found at Mercury. The main radiation source is the Sun which, due to it proximity to Mercury, is equivalent to 11 times as that seen from Earth. The Sun's heat would prohibit the operation of the spacecraft except that a dedicated sunshade is used to protect it at all times from direct illumination. The main concern for the GRS is Mercury itself, which has a surface temperature that can surpass $400{ }^{\circ} \mathrm{C}$. At periapsis (distance of closest approach) the spacecraft is only $200 \mathrm{~km}$ from the planet's surface, which occupies $\sim 1 / 3$ of the spacecraft's field of view. During the short time at this distance ( 30 minutes) the GRS experiences a very hot thermal transient and heats up significantly. Protecting the detector from these thermal pulses was the primary objective of the thermal design and testing program.

\section{A. MLI Blanket}

A sunshade is used to protect the spacecraft from direct illumination from the Sun. However, the spacecraft can not be similarly protected from Mercury's hot surface since many of the instruments on board must have a direct view of the planet. Therefore, a multi-layer insulation (MLI) blanket was used to limit the GRS from the intense thermal transients.

The MLI blankets consist of an outer layer of $25 \mu \mathrm{m}$ thick Kapton followed by 18 layers of double aluminized mylar $6 \mu \mathrm{m}$ thick, each separated by a layer of Dacron netting. The netting provides a good thermal barrier between adjacent mylar layers. 
The innermost layer is Dacron sail cloth, providing strength to the blanket. The thermal blankets entirely surround the GRS, except for small holes allowing for the support structures and passive radiator. The MLI blanket intercepts the $400{ }^{\circ} \mathrm{C}$ infrared radiation coming from the Mercury surface but causes no significant attenuation to the gamma rays.

\section{B. Passive Radiator}

The mechanical cooler uses up to $15 \mathrm{~W}$ input power, and the waste heat must be rejected. If not rejected efficiently, this heat would increase the GRS/cooler-body temperature causing the thermal burden on the detector to increase and the efficiency of the cooler to decrease. Therefore, a passive radiator was required to reject the waste heat. The radiator consists simply of a large aluminum plate that looks out at space. The surface of the radiator is clear anodized so that it has a low absorptivity but a high emissivity. As described below, there is a strong interaction between the infrared radiation coming from the planet surface, the radiator, and the GRS.

In a separate effort by one of the authors (B. Williams) the spacecraft thermal environment was used to model the external temperature extremes experienced by the GRS. Fig. 2 shows the change of temperature throughout the orbital period of the radiator, mechanical cooler, and GRS housing. Note the severe heat transient experienced as the spacecraft passes near the planet surface. It was found that the radiator can reach as high as $160{ }^{\circ} \mathrm{C}$. However, because of the thermal mass of the GRS, its temperature does not rise significantly until the spacecraft is passing away from the planet. Since the significant portion of data taken is only near the surface, any increase in detector temperature after that is inconsequential. During the remainder of the 12 hour period, the radiator again cools the system.

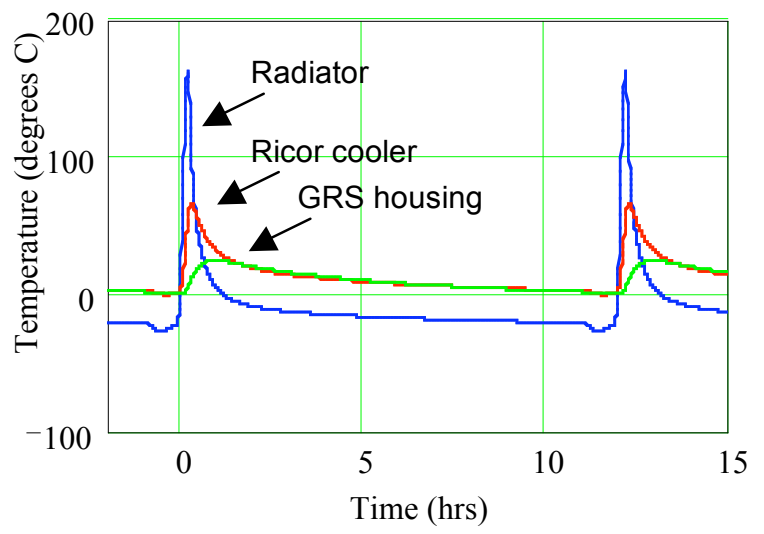

Fig. 2. Temperature profile across a representative orbit. The GRS experiences a large thermal transient when it passes near the planet every 12 hours. The radiator shows the largest temperature extreme. The cooler and GRS body respond more slowly to the heat pulse due to their thermal mass.

\section{Radiation Damage and Annealing Capability}

In space, energetic hadronic particles cause radiation damage in germanium detectors by creating large disordered regions [7][13]. These regions trap holes from the electron/hole pairs generated from gamma ray interactions. This trapping results in a degradation of energy resolution and a low side tailing in the line shape. The amount of degradation depends on the radiation fluence as well as the geometry and type of the detector (n-type or p-type). The fluence of damaging cosmic rays in space is $\sim 10^{8}$ $/ \mathrm{cm}^{2} /$ year. To minimize the effects of radiation damage, the detector should be kept as cold as possible, ideally below $85 \mathrm{~K}$. This strategy was employed successfully with the germanium detectors in both RHESSI (Reuven Ramaty High Energy Solar Spectroscopic Imager) and TGRS (Transient Gamma-Ray Spectrometer), providing excellent gamma-ray energy resolution for several years in space [4],[14].

However, the GRS will differ from TGRS and RHESSI in that it will not be possible to keep the detector cold during flight. This is due to the seven-year cruise time to Mercury and the limited lifetime of the cooler. In addition, the fluence of Solar energetic particles is highly unpredictable. Therefore, radiation damage is expected but can not be well characterized. Fortunately, this damage can be repaired by an annealing cycle. Annealing involves heating the detector to an elevated temperature and maintaining it there for a sufficient time. During the cruise a small heater will be used to maintain the detector at $300 \mathrm{~K}$. This modest temperature will allow for some annealing to occur continuously during the cruise. However, the time needed to fully recover a radiation damaged germanium detector is strongly dependent on temperature. Therefore, the system has the capability to heat the detector to as high as $358 \mathrm{~K}$. Because there is always some risk in taking the detector to an elevated temperature, the energy resolution will be monitored, and this higher temperature annealing cycle will only be performed as needed.

\section{THERMAL MOCKUP}

A complete mockup of the GRS was constructed for the purpose of testing the thermal performance of the system. The mockup consisted of a vacuum chamber and mechanical support as well as the radiation shields and detector encapsulation. The mockup allowed for the verification of the mechanical cooler performance, radiation shield performance, performance of the Kevlar stringing for conductive isolation, and determination of the thermal time constants of the system. Most importantly, it allowed for the determination of the absolute heat load on the system and the determination of the detector operating temperature under various conditions.

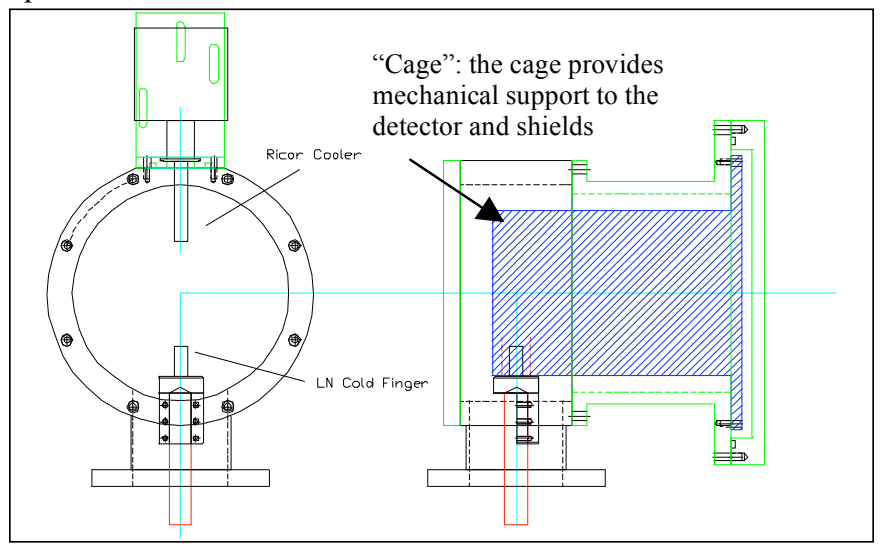

Fig. 3. Simplified drawing of the thermal mockup. The mockup has two cooling ports: one for the Ricor cooler and one for a liquid-nitrogen cold finger. The cage assembly is also shown. The detector is strung to the cage via the Kevlar suspension. The shields are supported by Vespel standoffs to the detector.

Fig. 3 shows a simplified drawing of the test setup. The setup consists of a vacuum chamber and mechanical support referred to as the cage. The cage holds the detector encapsulation via the Kevlar suspension. In place of the germanium crystal, the encapsulation contains a piece of brass machined to have a 
similar size and thermal mass. Several concentric radiation shields completely surround the encapsulation. The shields are held to the detector encapsulation using Vespel standoffs. The standoffs were machined to have minimal surface contact to limit the heat conduction between the shields. The vacuum housing has two ports. The first port is for the Ricor mechanical cooler. The second port allows for a liquid-nitrogen $\left(\mathrm{LN}_{2}\right)$ coldfinger assembly. In some cases it was useful to test with $\mathrm{LN}_{2}$ so that the properties of the system could be decoupled from the properties of the cooler. During testing, a copper braid was used to connect either the cooler or the $\mathrm{LN}_{2}$ cold finger to the encapsulation. The copper braid, identical to that used on the flight GRS, had a conductance $\mathrm{C}$ of $50 \mathrm{~mW} / \mathrm{K}$. Temperature sensors were placed on either end of the copper braid, that is, on the cold finger as well as on the encapsulation. The heat flow $\mathrm{Q}$ from the system to the cooler is then calculated using $Q=C^{*}$ $\Delta \mathrm{T}$.

\section{A. Radiation Shields}

A series of three thin aluminum cylinders, closed at each end, surround the detector and intercept the infrared radiation from the vacuum housing. These radiation shields are critical to the thermal performance of the system. Considerable effort was made to give them the lowest possible surface emissivity, as this determines the amount of infrared heat that will be transmitted from the shields to the detector. The shields received an opticalquality mechanical polish. They were then plated with $18 \mu \mathrm{m}$ of nickel and the surface was polished again. Finally, they were plated with $2.5 \mu \mathrm{m}$ of gold. The nickel plating is necessary for the gold to adhere to the aluminum surface. The surface emissivity was measured using an infrared laser at $1000 \mathrm{~nm}$ at room temperature, and a typical value of 0.02 was achieved.

It was not possible in our program to measure the surface emissivities directly at cryogenic temperatures. Furthermore, the limiting factor on the shield performance was not necessarily the surface finish. Rather, the limit was set by the fact that each shield had several small holes to allow for signal wires and the mechanical-cooler cold-finger to pass through. The holes act like "black" surfaces as far as infrared radiation is concerned. This is hard to quantify and thus the design required a "best effort" to minimize the number and size of the holes. Because of these two factors, it was important to verify the shield performance using the full thermal mockup.

For optimal performance, all other surfaces critical to radiation transfer were plated and polished as well. Primarily this included the detector encapsulation and the inside of the vacuum housing but also included various miscellaneous parts.

\section{B. Kevlar Suspension}

Because some structural attachment from the GRS housing to the detector is required, the connection provides a conductive path for heat to the detector. To minimize this path, the detector is suspended using a Kevlar fiber suspension scheme. The thermal conductivity of the fiber is $0.27 \mathrm{~W} / \mathrm{m}^{*} \mathrm{~K}$ (at $150 \mathrm{~K}$ ), providing good thermal isolation between the housing and the detector. The total heat conduction from the Kevlar fibers was calculated to be $\sim 5 \mathrm{~mW}$.

The signal wires from the detector also provided a heat path. The wires were $0.254-\mathrm{mm}$ diameter stainless steel and included the detector signal, high voltage, Zener heater, and temperature sensor connections. The total heat load was calculated to be $\sim 6$
$\mathrm{mW}$. Thus the total conductive load, $11 \mathrm{~mW}$, was small compared to the expected radiative load.

\section{TESTING AND RESULTS}

\section{A. Mechanical Cooler}

The cooler selected as meeting all the requirements (heat lift, lifetime, low power, and low mass) was a small Stirling-cycle cooler from Ricor (model K508). This device has a nominal heat lift of $750 \mathrm{~mW}$ at $80 \mathrm{~K}$ with an input power of $\sim 15 \mathrm{~W}$. This is specified at 40-bar helium fill pressure at $25{ }^{\circ} \mathrm{C}$ heat rejection temperature. Ricor coolers are intrinsically rugged and will withstand the harsh vibrations of the rocket launch. However, the lifetime of the cooler is an issue. The lifetime is known to depend in part on the fill pressure and can be extended by lowering the pressure. Because of the length of the mission (1 Earth year in orbit around Mercury) it was determined that operating at 30 bar would meet the desired safety margin for lifetime. The tradeoff is that lowering the fill pressure also reduces the heat lift. Therefore, several coolers were obtained at 30-bar fill pressure and, as part of the thermal feasibility test program, were fully characterized under a range of conditions.

The first tests involved mounting the coolers into an empty vacuum cryostat. A small copper block was used in place of the detector. Mounted on the block were a temperature sensor and a Zener heater for introducing a known heat load. Another temperature sensor was mounted directly onto the cryocooler cold-tip. This configuration allowed the coolers to be tested independently of the thermal properties of the GRS. Fig. 4 shows the equilibrium temperature achieved at the detector block as a function of thermal burden for two coolers. The results are shown at $10-\mathrm{W}$ and $13-\mathrm{W}$ input power to give a range of operation. At $85 \mathrm{~K}$ (the target GRS operating temperature) and $13-\mathrm{W}$ input power the cooler can lift $500 \mathrm{~mW}$ with 30-bar fill pressure.

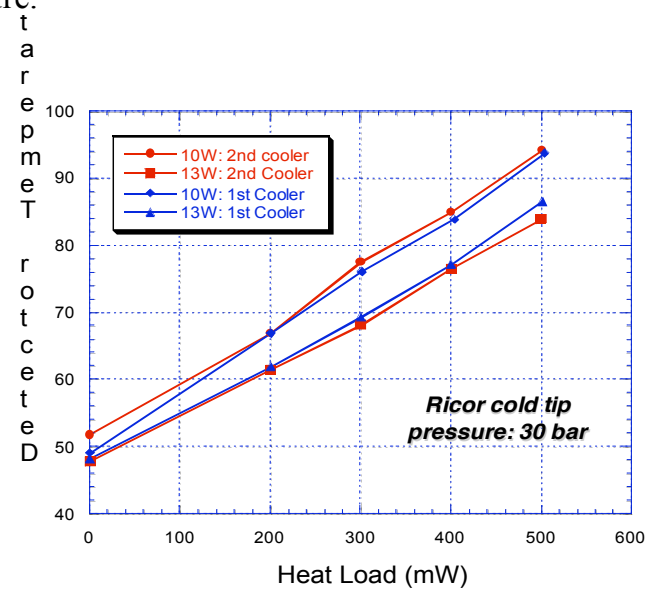

Fig. 4. Detector temperature vs. heat load. Two Ricor K508 coolers were characterized for their heat lift capability at 30-bar fill pressure. Heat lift is shown at 10 and $13-\mathrm{W}$ for comparison. The ambient temperature was $25^{\circ} \mathrm{C}$.

\section{B. GRS Thermal Mockup}

Once the heat lift capability of the mechanical coolers was determined, testing of the GRS thermal design began. It was of course necessary to ensure that the heat load of the GRS was less than the lift capability of the cooler (including a margin of safety) for the desired operating conditions. The full thermal prototype was mounted into the vacuum cryostat including cage, detector encapsulation and radiation shields. 
The total heat load was measured under the conditions expected during orbit. The system was tested at 25 and $41{ }^{\circ} \mathrm{C}$. These temperatures were chosen because $25{ }^{\circ} \mathrm{C}$ is the average temperature expected during the mission and $41{ }^{\circ} \mathrm{C}$ represents the extreme temperature that the GRS will reach during the hot thermal transients. The elevated temperature was achieved by wrapping the vacuum vessel in heat tape and letting it come to thermal equilibrium. The absolute heat load was determined by measuring the $\Delta \mathrm{T}$ across the cold-braid connecting the detector encapsulation to the cooler. The absolute head load was found to be $430 \mathrm{~mW}$ at $41{ }^{\circ} \mathrm{C}$.

For comparison, a test was also made with only one shield installed. This was done to better quantify the effectiveness of the shields and to benchmark the thermal simulations that were performed. Table 1 shows the some of these results for the total heat load under various conditions.

The total heat load is the sum of the conductive and radiative loads to the system. However, as described above, the conductive heat load was only $\sim 11 \mathrm{~mW}$ due to the effective isolation of the Kevlar suspension. Thus the majority of the load was due to radiative heating.

Table 1. GRS Mockup Heat Load

\begin{tabular}{||c|c|c|}
\hline \hline \# Shields & Ambient Temp. $\left({ }^{\circ} \mathrm{C}\right)$ & Total Heat Load $(\mathrm{mW})$ \\
\hline 3 & 41 & 430 \\
\hline 3 & 25 & 280 \\
\hline 1 & 25 & 500 \\
\hline
\end{tabular}

The most important test was to determine the detector operating temperature using the cooler under different conditions. Table 2 shows this temperature for $10-\mathrm{W}$ and $13-\mathrm{W}$ cooler power. Since the cooler efficiency depends heavily on the its rejection temperature (i.e., temperature of the GRS housing) the cryostat and cooler were heated to $41{ }^{\circ} \mathrm{C}$ for another set of measurements. It was found that even at the low power setting $(10 \mathrm{~W})$ at rejection temperature of $41{ }^{\circ} \mathrm{C}$, the cooler could still maintain the detector within the acceptable range of operating temperatures.

Table 2. Detector Equilibrium Temperature

\begin{tabular}{||c|c|c||}
\hline $\begin{array}{c}\text { Ricor Input } \\
\text { Power }(\mathrm{W})\end{array}$ & $\begin{array}{c}\text { Detector } \\
\text { Temp }(\mathrm{K})\end{array}$ & $\begin{array}{c}\text { Heat Sink } \\
\text { Temperature }\left({ }^{\circ} \mathrm{C}\right)\end{array}$ \\
\hline 10 & 74.9 & 25 \\
\hline 10 & 88.4 & 41 \\
\hline 13 & 66.1 & 25 \\
\hline
\end{tabular}

\section{Annealing Time Constants}

As discussed in the section on radiation damage, it is likely that one or more annealing cycles will be necessary during the mission. Since this represents a down time in which no data can be taken, the time constants involved need to be known for proper planning. The thermal time constants were measured for raising the temperature up to the annealing temperature $(358 \mathrm{~K})$ and as well as for cooling the system to operating temperature $(85 \mathrm{~K})$. Providing $2 \mathrm{~W}$ to the Zener heater, the annealing temperature could be reached in 15 hours. After that, $100 \mathrm{~mW}$ was sufficient to maintain the temperature. With the cooler at full power the detector cooled down from $300 \mathrm{~K}$ to $85 \mathrm{~K}$ in 28 hours.

The most favorable opportunity for performing the anneal will be during one of the eclipse seasons. Eclipse seasons are when the spacecraft spends much of its time eclipsed from the Sun by
Mercury and last about one week each. Since there will be very little solar power during this time, much of the instrumentation will be shut off. However, sufficient power should be available to run the annealing heater. Thus the annealing can be performed with minimum loss of data.

\section{Thermal Simulations}

Throughout the course of this work, finite difference analysis was used in conjunction with measurements to characterize the thermal aspects of the system. To this end the full GRS sensor was modeled. While some parts of the system were well characterized (such as the shields and encapsulation in which the surface emissivity was explicitly measured), it was not possible to measure the properties of many other parts (such as support structures, feedthroughs etc.) Therefore, the simulations were benchmarked against measurements of the full system. Table 3 shows the predicted performance of the shields after benchmarking.

Table 3. Performance of I.R. Shields

\begin{tabular}{||c|c|c||}
\hline \hline \# Shields & $\begin{array}{c}\text { Heat Load }(\mathrm{mW}) \\
\text { measured }\end{array}$ & $\begin{array}{c}\text { Heat Load }(\mathrm{mW}) \\
\text { predicted }\end{array}$ \\
\hline 3 & 280 & 280 (benchmark) \\
\hline 2 & - & 325 \\
\hline 1 & 500 & 476 \\
\hline 0 & - & 680 \\
\hline
\end{tabular}

\section{REFERENCES}

[1] Solomon, S. C., et al., The MESSENGER mission to Mercury: Scientific objectives and implementation, Planet. Space Sci., 49, pp. 1445-1465, 2001.

[2] Gold, R. E., et al., The MESSENGER mission to Mercury: Scientific payload,Planet. Space Sci., 49, pp. 1467-1479, 2001.

[3] Santo, A. G., et al., The MESSENGER mission to Mercury: Spacecraft and mission design, Planet. Space Sci., 49, pp. 1481-1500, 2001.

[4] D. M. Smith et al., "The RHESSI Spectrometer", Solar Physics, 210, pp.3366, 2002.

[5] C. P. Cork, D. A. Landis, P. N. Luke, N. W. Madden, D. F. Malone and R. H. Pehl, "Encapsulation of Germanium Detectors for Space Flight", IEEE Transactions Nucl. Sci., vol. 43, no. 3, pp.1463-1466, June 1996.

[6] Kevlar used: Dupont 29 pre-twisted/braided cord, uncoated (no wax), 12 strand 400 denier approximately $.7 \mathrm{~mm}$ diameter Tensile strength: (breaking) $200 \pm 10 \mathrm{lbs} . \quad \mathrm{K}$ factor: (conductivity $\mathrm{W} / \mathrm{m}^{\circ} \mathrm{K}$ ) $=0.27 @ 150$ ${ }^{\circ} \mathrm{K}$

[7] H. W. Kraner, C. Chaseman, and K. W. Jones, "Effects produced by fast neutron bombardment of $\mathrm{Ge}(\mathrm{Li})$ gamma ray detectors," Nucl. Instrum. and Methods, vol. 62, pp. 173-183, 1968.

[8] F. S. Goulding and R. H. Pehl, "A Survey of Radiation Damage in Semiconductor Detectors," IEEE Trans. Nucl. Sci., vol. NS-19, p. 91, 1972.

[9] J. W. Cleland and J. H. Crawford, "Radiation-induced disorder in semiconductors," Proc. Int. Cont. on Semiconductor Physics, Prague pp. 299-302, 1960.

[10] L. S. Darken, R. C. Trammel, J. W. Raudorf, R. H. Pehl, J. H. Elliot, "Mechanism for Fast Neutron Damage of Ge(HP) Detectors," Nucl. Instrum. and Methods, vol. 171, pp. 49-59, 1980.

[11] R. Trammell and F. J. Walter, "The Effects of Carrier Trapping in Semiconductor Gamma-Ray Detectors," Nucl. Instr. and Meth., vol. 76, pp. 317-321, 1969.

[12] T. W. Raudorf and R. H. Pehl, "Effect of Charge Carrier Trapping on Germanium Coaxial Detector Line Shapes," Nucl. Instrum. and Methods., vol. A255, pp. 538-551, 1987.

[13] R. H. Pehl, E. L. Hull, N. W. Madden, J. Xing, "Gamma-ray escape peak characteristics of radiation-damaged reverse-electrode germanium coaxial detectors," Nucl. Instrum. and Methods., vol. A381, no.1, pp. 97-102, 1996.

[14] P. Kurczynski, E. L. Hull, N. W. Madden, "Long-term radiation damage to a spaceborne germanium spectrometer," Nucl. Instrum. and Methods., vol. A431, no. 1-2, pp. 141-147, 1999. 